\title{
THERMODYNAMIC PARAMETERS OF END-PLATE CHANNEL BLOCKADE ${ }^{1}$
}

\author{
JAMES G. MCLARNON AND DAVID M. J. QUASTEL ${ }^{2}$ \\ Department of Pharmacology and Therapeutics, Faculty of Medicine, The University of British Columbia, \\ Vancouver, British Columbia, V6T 1W5, Canada
}

Received June 13, 1983; Revised November 14, 1983; Accepted November 15, 1983

\begin{abstract}
The reaction kinetics for drug blockade of postsynaptic channels at the mouse neuromuscular junction were studied. The activation energies associated with the onward and off-rate constants, from a sequential model, were measured for procaine, scopolamine, octanol, and heptanol, and from these the transition state thermodynamic parameters of free energy $(\Delta \mathrm{G} \ddagger)$, enthalpy $(\Delta \mathrm{H} \ddagger)$, and entropy $(\Delta \mathrm{S} \ddagger)$ of activation were determined. All agents showed positive entropies of activation for the channel blocking rate constant $k_{2}$, with the values for the alcohols significantly greater than those for the two positively charged local anesthetics. No significant differences in the activation energies for the off-rate constant $k_{-2}$ were observed between any of the agents. The magnitude of $k_{-2}$ decreased as the hydrophobicity of the agent increased. The large activation energies measured for $k_{2}$ and $k_{-2}$ (in excess of $10 \mathrm{kcal} / \mathrm{mol}$ ) are higher than expected for simple rate-limiting diffusion, and the possibility exists that channel blockade involves conformational changes in a protein segment, induced by a hydrophobic interaction between agent and intrachannel site or sites. The large entropy increase observed in the blocking step is indicative of the agent replacing structured waters of hydration near a hydrophobic region of a protein.
\end{abstract}

A number of diverse agents cause the decay of miniature end-plate currents (MEPCs) to be divided into two exponential components. Considerable evidence has been presented which indicates that a sequential model can account for the biphasic MEPCs observed. The faster than normal component is due to "blockade" or "plugging" by the agent of ionic channels opened as a consequence of the combination of acetylcholine (ACh) with receptors, whereas the slower than normal component results from oscillation of the channel between open and "blocked" states (Adams, 1976, 1977; Pennefather and Quastel, 1980). The on- and off-rate constants, $k_{2}$ and $k_{-9}$, for "blocking" of end-plate channels by positively charged agents (but not agents presumed to act in the neutral form) are sensitive to transmembrane potential (Pennefather and Quastel, 1980) and to raised concentration of $\mathrm{Mg}^{2+}$ or $\mathrm{Ca}^{2+}$ ions (McLarnon and Quastel, 1983).

In the present study, we have examined the tempera-

${ }^{1}$ This work was supported by grants from the Muscular Dystrophy Association of Canada and the British Columbia Health Care Research Foundation. We thank Dr. J. Benbasat, Department of Biochemistry, The University of British Columbia, for helpful comments regarding the manuscript.

${ }^{2}$ To whom correspondence should be addressed. ture dependence of $k_{2}$ and $k_{-2}$ for a group of agents which cause biphasic MEPCs, namely, the aliphatic alcohols $n$ octanol and $n$-heptanol, the local anesthetic procaine, and scopolamine, which, at the concentration employed $(100 \mu \mathrm{M})$, acts like a local anesthetic on end-plate channels. Analysis, in terms of the thermodynamic parameters of rate theory, indicates that the "blocking" of end-plate channels by these agents is determined by a rate-limiting entropy-driven step, indicating hydrophobic interaction between agent and site(s). The measured activation energies for channel blockade by all agents (in excess of $11 \mathrm{kcal} / \mathrm{mol}$ ) were higher than expected for a diffusion-limited process, and for the two alcohols they were significantly greater than for the two positively charged agents. The activation energies for $k_{-2}$ were essentially the same for all of the agents, with the magnitude of $k_{-2}$ dependent upon the hydrophobicity of the agent. The large activation energies associated with $k_{2}$ and $k_{-2}$ could be due to the agents causing conformational changes in an interfacial hydrophobic region of a protein molecule.

\section{Materials and Methods}

The preparation used was the mouse diaphragm; MEPCs recorded from voltage-clamped end-plates were recorded in the temperature range from $8^{\circ} \mathrm{C}$ to $25^{\circ} \mathrm{C}$ and 
stored digitally on magnetic tape (Linder and Quastel, 1978; Pennefather and Quastel, 1980). The apparatus included the superperfusion system described by Cooke and Quastel (1973) which allowed the application of drugs to the end-plate within seconds and a temperature cooling system incorporating a Peltier device with electronic feedback control.

The solutions used contained $150 \mathrm{~mm} \mathrm{Na}, 5 \mathrm{mM} \mathrm{K}^{+}$, $2 \mathrm{mM} \mathrm{Ca}^{2+}, 1 \mathrm{mM} \mathrm{Mg}^{2+}, 24 \mathrm{mM} \mathrm{HCO}_{3}^{-}$buffer, $2 \times 10^{-8}$ $\mathrm{gm} / \mathrm{ml}$ of tetrodotoxin, and $2 \mathrm{gm} /$ liter of glucose equilibrated with $95 \% \mathrm{O}_{2}$ and $5 \% \mathrm{CO}_{2} . \mathrm{NO}_{3}{ }^{-}$salts were used to improve the signal to noise ratio of MEPCs (Linder and Quastel, 1978). In all experiments, paraoxon was used ( $4 \mu \mathrm{M}$ applied for $4 \mathrm{~min}$, then washed off) in order to prolong MEPCs. Poisoning of acetylcholinesterase results in a better definition (and computer fit) for the slow phase of the biphasic MEPC. The magnitudes of the onward and off-rate constants $\left(k_{2}\right.$ and $\left.k_{-2}\right)$ are unaffected by the treatment with paraoxon (Pennefather and Quastel, 1980; McLarnon and Quastel, 1983).

The methods used for averaging of MEPCs and subsequent analysis have been described previously (Pennefather and Quastel, 1981; McLarnon and Quastel, 1983). Briefly, parameters describing the time course of decay were determined from means of 25 to 40 individual MEPCs, aligned with respect to the point of maximal cross-correlation with a ramp function $(0,0 . .0,1,2)$, and averaged "off-line" after careful exclusion of "unusual" MEPCs, artifacts, etc. It should be noted that in the present experiments, using "channel-blocking" agents, MEPCs recorded after poisoning of $\mathrm{AChE}$ did not show the large diversity in decay rates observed in the absence of channel blocker, i.e., the averages of MEPCs closely represent individual MEPCs. To obtain the fitting parameters for the model (i.e., $k_{1}, k_{2}, k_{-2}, k_{3}$ ), the decay phase of the MEPC was characterized as the sum of two exponential components, after digital correction for recording filter and "deconvolution" of the MEPC using a 0.16 -msec time constant, to eliminate components related to asynchrony in channel opening (Linder et al., 1983).

The rate constants $\left(k_{2}\right.$ and $k_{-2}$ ) for end-plate channel blocking and unblocking were determined assuming a cyclic model as shown:

$$
\begin{array}{cc}
2 \mathrm{ACh}+R+D \underset{k_{-1}}{\stackrel{k_{1}}{\rightleftharpoons}} \mathrm{ACh}_{2} R^{*}+D \\
k_{4} 11 k_{-4} & k_{-2} 1 l k_{2} \\
2 \mathrm{ACh}+R D & \underset{k_{3}}{\stackrel{k_{-3}}{\rightleftharpoons}} \mathrm{ACh}_{2} R D
\end{array}
$$

where the states $\mathrm{ACh}_{2} R^{*}$ and $\mathrm{ACh}_{2} R D$ represent the open and the open-blocked channels, respectively. The conversion to another form from $\mathrm{ACh}_{2} R D$, other than the open channel, is introduced for two reasons: $(a)$ to account for a decrease in the total MEPC area with little or no change in peak amplitude caused by some of these blocking agents (Pennefather and Quastel, 1980; McLarnon and Quastel, 1983), and (b) without this step the normal voltage dependence of $k_{-1}$ can appear to be diminished or reversed by some channel blockers (Pennefather and Quastel, 1980; McLarnon and Quastel, 1983).
The rate constant $k_{-3}$ must be small since no triphasic decays (with low tail components) were observed. The $R$ $+D \rightleftharpoons R D$ step corresponds to block of closed end-plate channels (Adams, 1977). However, an appreciable ratio $k_{-4}[D] / k_{4}$ would be manifest in a reduction of initial (peak) MEPC height, which was not a prominent feature in the present results. The rate constants $k_{2}, k_{-2}$, and $k_{3}$ can be determined from kinetic analysis of the decay phase of the MEPC, on the assumption that the normal channel-closing rate constant $k_{-1}$ is not altered by the agent and that $k_{-3}, k_{-4}$, and $k_{4}[R D]$ are negligible during the decay of the MEPC. A least squares fitting program determined the slopes and intercepts of the fast and slow components of the MEPC (and the area under the MEPC), and from these the rate constants $k_{2}, k_{-2}$, and $k_{3}$ were calculated using the equations given in $\mathrm{Mc}$ Larnon and Quastel (1983). Since the values of $k_{3}$ were generally small for the agents studied $\left(<0.4 \mathrm{msec}^{-1}\right)$, the values of $k_{2}$ and $k_{-2}$ given under "Results" are close to the values calculated using a simple sequential model with $k_{i 3}$ and $k_{-4}$ set to zero.

The procedures followed in the thermodynamic analysis are outlined in Minneman et al. (1980) (see also Weiland and Molinoff, 1981). The transition state activation energy $E_{a}$ was determined from the slope of an Arrhenius plot [ $\ln$ (rate constant) versus $T^{-1}$ ] for both the rate constants for channel blocking $\left(k_{2}\right)$ and unblocking $\left(k_{-2}\right)$. The enthalpy and free energy of activation for the transition state were then found from $\Delta \mathrm{H} \ddagger=E_{a}-$ $R T$ and $\Delta \mathrm{G} \ddagger=-R T \ln k_{r}+R T \ln k T / h$, respectively, where $k_{r}$ is the rate constant, $k$ is Boltzmann's constant, and $h$ is Planck's constant. The entropy of activation $(\Delta \mathrm{S} \ddagger)$ is then found using: $\Delta \mathrm{G} \ddagger=\Delta \mathrm{H} \ddagger-\mathrm{T} \Delta \mathrm{S} \ddagger$. Values for enthalpy $\left(\Delta \mathrm{H}^{\circ}\right)$, entropy $\left(\Delta \mathrm{S}^{\circ}\right)$, and free energy of activation $\left(\Delta \mathrm{G}^{\circ}\right)$ for the $\mathrm{ACh}_{2} R D$ state ("associated state") were then derived by subtracting the channelunblocking transition state parameters from the channel-blocking values. The associated state parameters are equilibrium values if the same rate-limiting step underlies the channel-blocking and -unblocking processes.

To obtain means and SEMs for thermodynamic parameters, estimates were made individually for each junction where MEPCs were recorded at four or more specified temperatures.

\section{Results}

Figure 1 shows the modification of the time course of MEPCs with variation of temperature in the presence of a constant concentration of a drug. In each case, the data are from a single junction and each average is of 25 to 40 MEPCs. It is clear from Figure 1 that both components of the MEPC decay phase are sensitive to temperature for all of the agents.

The obvious differences in time course of the MEPCs, with different drugs and with varied temperature, reflect differences in the values of $k_{2}[D]$ and $k_{-2}$ (Table I). For example, the large slow phase with scopolamine reflects a relatively high ratio of $k_{-2}$ to $k_{2}[D]$, and the rise in the slow phase (relative to the fast phase) with lowering of temperature reflects a higher $Q_{10}\left(\right.$ and $E_{a}$ ) for $k_{2}[D]$ than for $k_{-2}$. Figure $2 A$ shows Arrhenius plots for the averages of the channel-"blocking" rate constant $\left(k_{2}\right)$ for the four 

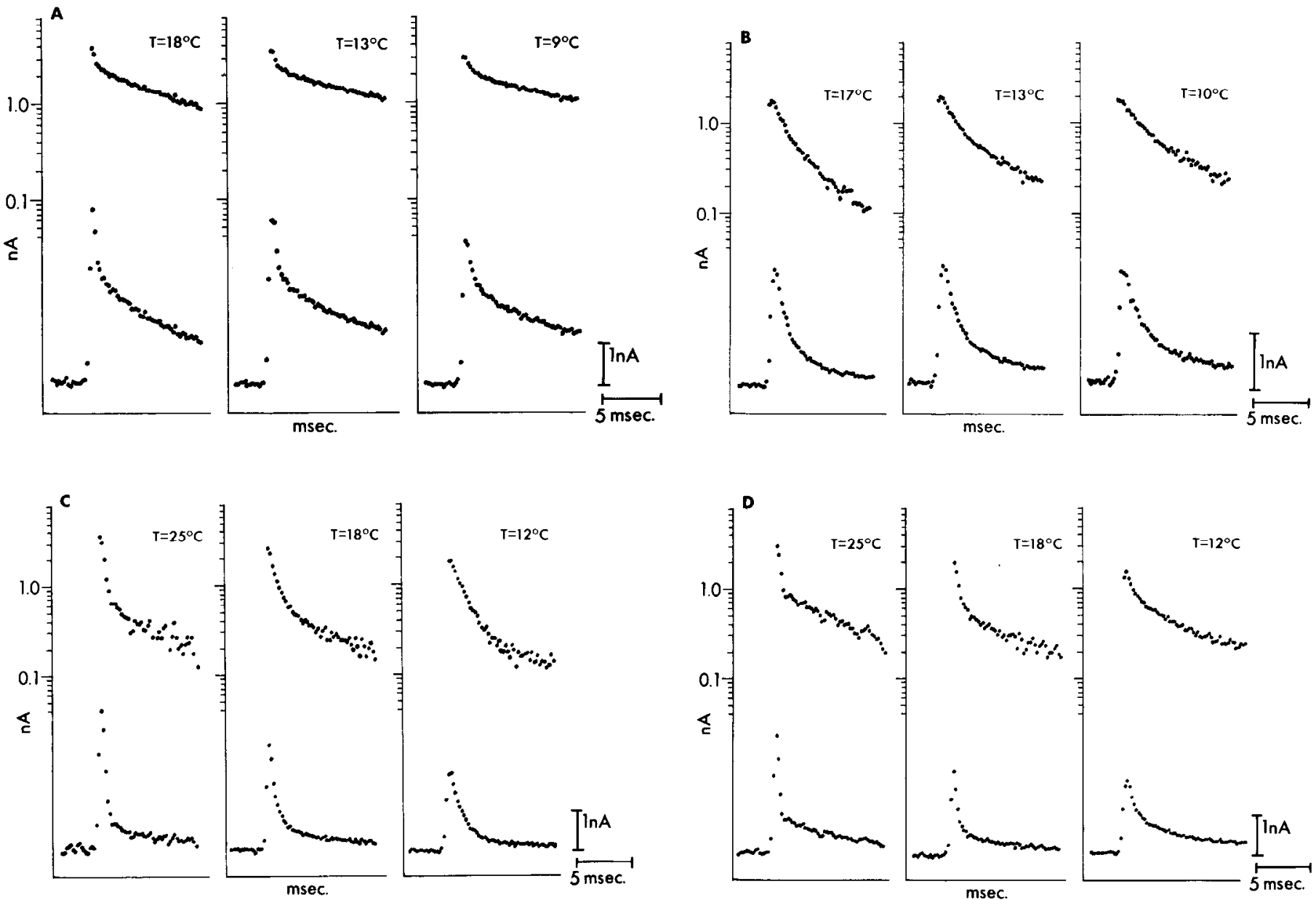

Figure 1. MEPCs plotted linearly (lower traces) and semilog (upper traces) at $-80 \mathrm{mV}$. A, Scopolamine (100 $\mu \mathrm{M}) ; B$, Procaine $(25 \mu \mathrm{M}) . C$, Octanol $(100 \mu \mathrm{M}) . D$, Heptanol $(250 \mu \mathrm{M})$. Data for each agent were obtained from the same cell at the temperatures shown. Each plot represents the average of 25 to 40 MEPCs.

TABLE I

Thermodynamic parameters associated with channel blocking and unblocking as determined from transition state analysis Associated state values were calculated from the differences between the transition state parameters for channel blocking and unblocking. Rate constants are values for $T=25^{\circ} \mathrm{C}$ and $\mathrm{V}=-80 \mathrm{mV}$. Each tabulated value is mean \pm SEM of values obtained separately for each end-plate studied.

\begin{tabular}{|c|c|c|c|c|c|c|c|}
\hline Agent & $\begin{array}{c}\text { No. of } \\
\text { End-plates }\end{array}$ & $\begin{array}{c}k_{2}\left(10^{6} \mathrm{M}^{-1} \mathrm{sec}^{-1}\right) \\
\text { or } \\
k_{-2}\left(\mathrm{msec}^{-1}\right)\end{array}$ & $E_{a}$ & $\Delta \mathrm{H}^{+}$ & $\Delta \mathrm{G} \ddagger$ & $\mathrm{T} \Delta \mathrm{S} \ddagger$ & $\Delta S \ddagger$ \\
\hline & & & $k c a l / m o l$ & $\mathrm{kcal} / \mathrm{mol}$ & $k c a l / m o l$ & $k c a l / m o l$ & entropy units \\
\hline Procaine & 6 & $29.7 \pm 2.9$ & $12.0 \pm 0.9$ & $11.4 \pm 0.9$ & $7.3 \pm 0.2$ & $4.1 \pm 0.4$ & $13.8 \pm 1.5$ \\
\hline Scopolamine & 3 & $18.2 \pm 2.0$ & $11.6 \pm 1.3$ & $11.0 \pm 1.2$ & $7.6 \pm 0.2$ & $3: 5 \pm 0.5$ & $11.7 \pm 1.8$ \\
\hline Octanol & 5 & $17.4 \pm 0.9$ & $18.4 \pm 0.7$ & $17.8 \pm 0.7$ & $7.6 \pm 0.2$ & $10.2 \pm 0.7$ & $34.2 \pm 2.7$ \\
\hline Procaine & 6 & $0.77 \pm 0.05$ & $10.0 \pm 0.6$ & $9.4 \pm 1.0$ & $13.5 \pm 0.5$ & $-4.1 \pm 0.6$ & $-13.8 \pm 2.2$ \\
\hline Scopolamine & 3 & $2.36 \pm 0.17$ & $10.3 \pm 0.9$ & $9.7 \pm 0.8$ & $12.9 \pm 0.5$ & $-3.2 \pm 0.4$ & $-10.7 \pm 1.4$ \\
\hline Octanol & 5 & $0.55 \pm 0.05$ & $10.4 \pm 0.8$ & $9.8 \pm 0.8$ & $13.7 \pm 0.5$ & $-3.9 \pm 0.4$ & $-13.1 \pm 1.4$ \\
\hline Heptanol & 5 & $1.01 \pm 0.07$ & $10.6 \pm 0.7$ & $10.0 \pm 0.7$ & $13.4 \pm 0.5$ & $-3.4 \pm 0.4$ & $-11.4 \pm 1.4$ \\
\hline Associated state & & & & $\Delta H^{\circ}$ & $\Delta G^{\circ}$ & $T \Delta S^{\circ}$ & $\Delta S^{\circ}$ \\
\hline Procaine & 6 & & & 2.0 & -6.2 & 8.2 & 27.6 \\
\hline
\end{tabular}



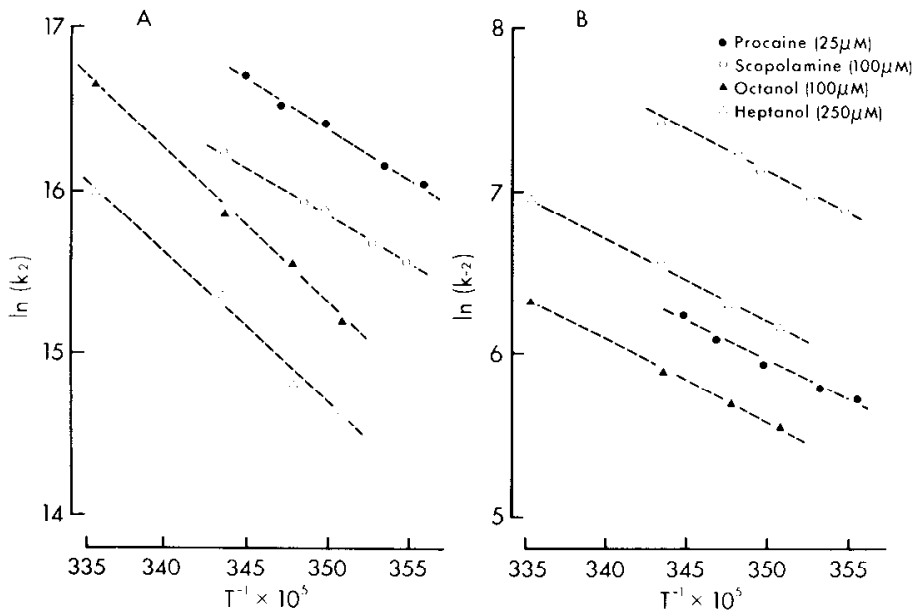

Figure 2. Arrhenius plots for the rate constants $k_{2}$ and $k_{-2}$ in the sequential model. $A$, Channel block $\left(k_{2}\right) . B$, Channel unblock $\left(k_{-2}\right)$. The fits to the data were determined by linear regression analysis. Plotted points are means, from all junctions studied, each with a standard error of about $0.2 \log _{e}$ unit.

agents studied and fits to these data using linear least squares regression. The activation energy $E_{a}$ for channel block is equal to the slope of the Arrhenius plot multiplicd by the universal gas constant $R$; the values of $E_{a}$ obtained from the averages of $k_{2}$ were the same as those given in Table I, where the values are the means of $F_{a}$ for individual junctions. For procaine and scopolamine, the activation energies for channel blocking were in the range of 11 to $12 \mathrm{kcal} / \mathrm{mol}\left(Q_{10}\right.$ values about 1.8 for the temperature range $20^{\circ} \mathrm{C}$ to $10^{\circ} \mathrm{C}$ ), whereas for the two aliphatic alcohols, octanol and heptanol, activation energies were significantly higher, in the vicinity of $18 \mathrm{kcal} /$ $\mathrm{mol}\left(Q_{10}>2\right)$. Gage et al. (1978) found activation energies of $11.3 \mathrm{kcal} / \mathrm{mol}$ and $18.2 \mathrm{kcal} / \mathrm{mol}$ at two end-plates for a single phase of decay, with $1 \mathrm{~mm}$ octanol, at the frog neuromuscular junction. It is likely that the inverse of their single time course corresponds to $k_{2}[D]$ in the present work since the initial decay of a MEPC in the presence of a channel blocker is given by $k_{2}[D]+k_{-1}$ which, with octanol block, is dominated by the first term. It should be noted that the slow component for octanol has a low amplitude (Pennefather and Quastel, 1980) and can easily be obscured by baseline noise if high concentrations of octanol are used, giving a value of $k_{2}[D]$ that is high relative to $k_{-2}$.

The Arrhenius plots for the channel-blocking rate constant $k_{-2}$ are shown in Figurc $2 B$. Activation energies were determined in the same manner as for $k_{2}$ and are listed in Table I. The results show activation energies of about $10 \mathrm{kcal} / \mathrm{mol}$ with no significant differences between any of the four agents studied.

The transition rate theory parameters for free energy of activation, enthalpy, and entropy for channel blocking and unblocking (determined from the equations given under "Materials and Methods") are also included in Table I. The results, for the rate constant $k_{2}$, show that the enthalpies of activation and entropies of activation are substantially higher for the two alcohols, octanol and heptanol, than for the two local anesthetics, procaine and scopolamine. The very similar activation energies for octanol and heptanol action occur despite a 2 -fold difference in their rate constants, with the higher rate constant corresponding to the more hydrophobic of the two, octanol. It is also notable that in each case both the enthalpy and the entropy change is positive, i.e., the channel-blocking step is "entropy driven," which also suggests the participation of hydrophobic bond formation (Kauzmann, 1959).

No significant differences in the activation energies associated with the rate constant of channel unblocking $\left(k_{-2}\right)$ were observed for the four agents studied. However, the similarity in the activation energies for the alcohols was again associated with a substantial difference in their rate constants as the octanol $k_{-2}$ value was about onehalf the corresponding value for heptanol. Thus, the more hydrophobic agent, octanol, is stabilized at the binding site, relative to heptanol. The magnitude of $k_{\cdots \cdot 2}$ decreased in the order of increased hydrophobicity of agent, after correcting for the voltage dependence of $k_{-2}$ for procaine and scopolamine action (Pennefather and Quastel, 1980).

The thermodynamic parameters for the associated state can be derived from the transition state parameters (see "Materials and Methods") assuming that the same rate-limiting step governs the kinetics for the on- and off-rate constants. The data in Table I show reaction negative-free energies $\left(\Delta \mathrm{G}^{\circ}\right)$, positive enthalpies $\left(\Delta \mathrm{H}^{\circ}\right)$, and positive entropies $\left(\Delta S^{\circ}\right)$. The profiles for the various thermodynamic parameters are shown for octanol and procaine in Figure 3 as the dissociated state proceeds to an associated state through the formation of an activated complex. The free energy profiles for the two agents are similar, whereas their respective enthalpy and entropy plots are markedly different for both the transition state and the associated state.

It should be noted that temperature changes can alter
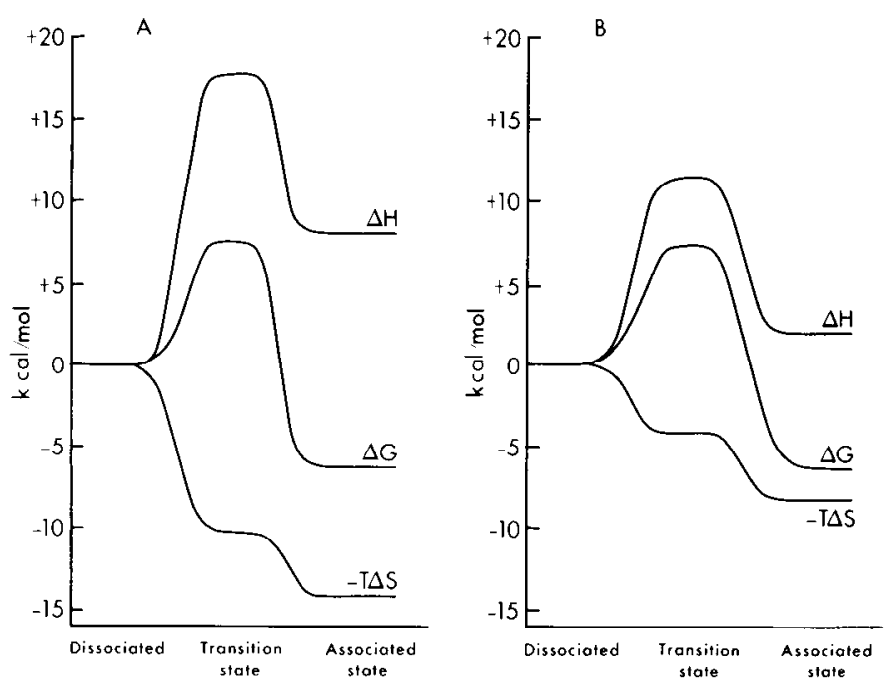

Figure 3. Profiles of thermodynamic parameters for enthalpy $(\Delta \mathrm{H})$, free energy $(\Delta \mathrm{G})$, and temperature $\times$ entropy $(-\mathrm{T} \Delta \mathrm{S})$ for octanol $(A)$ and procaine $(B)$. 
the degree of ionization for charged agents, which could, in turn, affect $k_{2}$ or $k_{-2}$. However, it is likely that any changes in the degree of ionization would be insignificant in the present work, since the temperature changes involved are relatively small and the Arrhenius plots are linear.

\section{Discussion}

In this study we have applied kinetic rate theory to the action of certain drugs to block end-plate channels (Adams, 1976). On this basis, the pseudo-first-order rate constant $k_{2}[D]$ in the cyclic model represents the binding of the agent lo some site once a channel has been opened, and the rate constant $k_{-2}$ represents channel unblocking, which presumably involves the dissociation of the blocking agent from its binding site.

The results show that for all of the agents studied, channel blockade involves large positive entropy and enthalpy changes in the formation of a transition state or "activated complex." Similar increases in entropy have been observed in other systems (e.g., Minneman et al., 1980) and attributed to the formation of intermolecular hydrophobic bonds (cf. Kauzmann, 1959; Tanford, 1980). Data in Table I also point to the involvement of hydrophobic bonds in the interaction of the agents with the end-plate channel. For the onward or blocking rate constant $k_{2}$, the alcohols, octanol and heptanol, have equal activation energies $E_{a}$ (within experimental error), although the rate constant for channel block for the more hydrophobic agent, octanol, is almost twice that for heptanol. In addition, the activation energies associated with the channel-unblocking step are also very similar for the two alcohols, although the $k_{-2}$ value for octanol is less than one-half that for heptanol. A clear correlation between the degree of hydrophobicity of the agents and $k_{-2}$ is evident from Table I; as lipid solubility of the agent increases, the magnitude of $k_{-2}$ is diminished. The thermodynamic parameters for the association of agent with site show large positive entropy changes and smaller positive enthalpy changes, indicating that nonhydrophobic interactions are not significant. The driving force for the overall process (negative free energy, $\Delta \mathrm{G}^{\circ}$ ) is the large increase in entropy, $\Delta \mathrm{S}^{\circ}$.

The activation energies measured for the rate constants $k_{22}$ and $k_{-2}$ are higher than would be expected for simple diffusion in an aqueous medium, but it is conceivable that the high activation energies found here could be related to a high temperature sensitivity of the topography of the channel, leading to a correspondingly high temperature sensitivity of the rate of diffusion of the drugs to and from their site(s) of action. Alternatively, the high activation energies may reflect drug-induced conformational changes (unfolding and folding, respectively) of an interfacial region of a protein molecule. The unfolding step would be accompanied by a positive entropy change for the onward rate constant $\left(k_{2}\right)$, as is observed. Similarly, the folding of the molecule to a more ordered form would be accompanied by a negative entropy change, as is observed for $k_{-2}$. The similar activation energies for $k_{-2}$ with all of the agents studied are consistent with a common site of action. If this is so, apparent lack of voltage dependence of $k_{2}$ and $k_{-2}$ for agents acting in a neutral form (Adams, 1976; Pennefather and Quastel, 1980) would imply that the conformational changes are voltage independent. The voltage dependence of the rate constants, for agents acting in the positive form, could then arise if the conformational change included the protein segment and bound agent. An increase in transmembrane potential could also act to diminish $k_{-2}$ by stabilization of attachment of a positively charged molecule at the site of interaction and increase $k_{2}$ by increasing the access of the molecule to the site.

It is of interest that the enthalpies in Table I, determined from the activated state complex for the on-rate constant $k_{2}$, are significantly higher for both alcohols than for procaine or scopolamine. This could arise if the interaction sites for the two groups were different, or, if diffusion of the drug to its site of action was rate limiting, it could reflect different pathways of action (i.e., nonpolar molecules acting from within the lipid phase). Alternatively, the differences in enthalpies could reflect a contribution of polar interactions between the positively charged local anesthetics and the channel site or its environment. The formation of ionic bonds would decrease the transition state enthalpy and contribute to the blocking action for these agents.

It is likely that the end-plate channel, for the most part, is a large aqueous pore (Linder and Quastel, 1978; Adams et al., 1980), and it is plausible that the environment of the blocking sites is polar (McLarnon and Quastel, 1983). Thus, one can imagine the hydrophobic region of the agent "escaping" the energetically unpreferred hydrophilic environment of the channel by associating with a hydrophobic interfacial region of the receptorchannel protein. The consequent replacement of some of the structured water of hydration around the site by the hydrophobic portion of the drug would result in an increase in the disorder of the system and possible localized conformational change(s) in the protein molecule. A conformational change in the receptor is also suggested by the sequential model itself, in which the transmitter (ACh) must remain attached to the receptor for as long as the blocking agent is attached to the receptor-channel site (Adams, 1976; Pennefather and Quastel, 1980). Whatever the actual mechanism of channel "blockade" by drugs, it is apparent that the kinetic rate constants for the drug effects are strongly dependent upon a hydrophobic interaction between agent and channel. Previous studies at end-plates have also shown the presence of hydrophobic effects for organic cations (Adams et al., 1981), $n$-alkyl guanidine derivatives (Farley et al., 1981), and atropine and scopolamine (Adler et al., 1978; McLarnon and Quastel, 1983).

\section{References}

Adams, D. J., T. M. Dwyer, and B. Hille (1980) The permeability of endplate channels to monovalent and divalent metal cations. J. Gen. Physiol. 75: 493-510.

Adams, D. J., W. Nonner, T. M. Dwyer, and B. Hille (1981) Block of endplate channels by permeant cations in frog skeletal muscle. J. Gen. Physiol. 78: 593-615.

$\Lambda$ dams, P. R. (1976) Drug blockade of open endplate channels. J. Physiol. (Lond.) 260: 531-552. 
944

McLarnon and Quastel

Vol. 4, No. 4, Apr. 1984

Adams, P. R. (1977) Voltage jump analysis of procaine action at frog endplates. J. Physiol. (Lond.) 268: 291-318.

Adler, M., E. X. Albuquerque, and F. J. Lebeda (1978) Kinetic analysis of endplate currents altered by atropine and scopolamine. Mol. Pharmacol. 14: 514-529.

Cooke, J. D., and D. M. J. Quastel (1973) Transmitter release by mammalian motor nerve terminals in response to focal polarization. J. Physiol. (Lond.) 228: 377-405.

Farley, J., J. Z. Yeh, S. Watanabe, and T. Narahashi (1981) Endplate channel block by guanidine derivatives. J. Gen. Physiol. 77: 273-293.

Gage, P. W., R. N. McBurney, and D. Van Helen (1978) Octanol reduces end-plate channel lifetime. J. Physiol. (Lond.) 274: 279-298.

Kauzmann, W. (1959) Some factors in the interpretation of protein denaturation. Adv. Protein Chem. 14: 1-63.

Linger, T. M., and D. M. J. Quastel (1978) A voltage clamp study of the permeability change induced by quanta of transwitter at the mouse endplate. J. Physiol. (Lond.) 281: 535556.

Linger, T. M., P. Pennefather, and D. M. J. Quastel (1983) The time course of miniature end-plate currents and its modification by receptor blockade and ethanol. J. Gen. Physcol., in press.

McLarnon, J., and D. M. J. Quastel (1983) Postsynaptic effect of magnesium and calcium at the mouse neuromuscular junction. J. Neurosci. 3: 1626-1633.

Minneman, K. P., G. A. Welland, and P. B. Molinoff (1980) A comparison of the beta-adrenergic receptor of the turkey erythrocyte with mammalian beta ${ }_{1}$ and beta $_{2}$ receptors. Mol. Pharmacol. 17: 1-7.

Pennefather, P., and D. M. J. Quastel (1980) Actions of anaesthetics on the function of nicotinic acetylcholine receptors. In Molecular Mechanisms of Anesthesia, B. R. Fink, ed., Vol. 2, pp. 45-58, Raven Press, New York.

Pennefather, P., and D. M. J. Quastel (1981) Relation between subsynaptic receptor blockade and response to quantal transniter at the mouse neuromuscular junction. J. Gen. Physiol. 78: 313-344.

Tanford, C. (1980) The Hydrophobic Effect, Ed. 2, J. Wiley \& Sons, New York.

Weiland, G. A., and P. B. Molinoff (1981) Quantitative analysis of drug-receptor interactions: Determination of kinetic equilibrium properties. Life Sci 29: 313-330. 